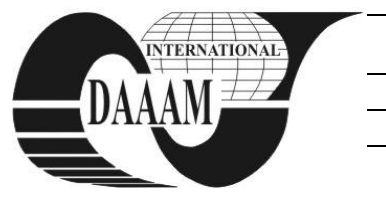

\title{
EXPERIMENTAL MACHINE TOOL FOR PROCESS MONITORING AND CONTROL SYSTEMS RESEARCH
}

\author{
STAROVESKI, T[omislav]; BREZAK, D[anko]; UDILJAK, T[oma] \& MAJETIC, D[ubravko]
}

\begin{abstract}
This paper presents the main characteristics of the 3axis milling machine retrofitted with the Linux-based CNC open architecture control system. It is developed on the basis of a technologically outdated industrial planing machine, which was redesigned for the purpose of the technological project "Intelligent Monitoring of a Machine Tool Main Spindle". The new machine is intended to be used for industrial, research and educational purposes, specifically, in the design and analysis of machining process monitoring and control algorithms.
\end{abstract}

Key words: machine tool retrofitting, open control systems, enhanced machine controller, experimental setup

\section{INTRODUCTION}

In this paper, characteristics of a machine tool controlled by the real-time open architecture control (OAC) system are presented. The machine was developed on the basis of technologically outdated industrial planing machine, which was retrofitted for the purpose of the technological project "Intelligent Monitoring of a Machine Tool Main Spindle". The primary aim of this project was to develop an industry applicable advanced motor spindle unit with monitoring and diagnostic system which could identify or predict motor spindle failures and tool breakage locally and remotely (LAN, TCP/IP protocol). For that purpose, a testbed platform had to be built in the form of a fully redesigned and $\mathrm{CNC}$ retrofitted 3-axis milling machine with OAC system (Pritschow, 2001). This machine could then be used in the analysis of different control and process monitoring algorithms and hardware modules, which was the second project objective. Among several advanced, continuously developing and currently most applicable OAC solutions (Asato et al., 2002), the oldest one, named as Enhanced Machine Controller (EMC), was implemented (Proctor \& Michaloski, 1993).

The basic characteristics of the machine tool structure design, main spindle unit and control system are given in the following sections together with the concluding remarks.

\section{THE MAIN DESIGN CHARACTERISTICS}

Large planing machine, type "HJ8A/2m" manufactured by "Kovosit Holoubkov" Co. in 1959, was used in the development of the milling testbed platform. Machine main drive was based on a simple $7.5 \mathrm{~kW}$ hydraulic system, where hydraulic cylinder was used for the main motion. Asynchronous motors with trapezoidal screw assemblies served as feed drives. In order to achieve linear relative motion, very simple control circuit existed, consisting only of a few reversing contactors and limit switches. Selected machine was considered a suitable base, because its support elements and guides provide sufficient rigidity for medium and light machining. However, extensive redesign of the most remaining parts was mandatory in order to achieve open CNC reconfigurable machine tool. Original machine configuration together with the redesigned form is shown in Figure 1.
Main spindle unit type HSM105SE-V01-FK719-11 produced by HSTec Co. is partly reconfigured in order to be upgraded with the spindle monitoring system made by Dittel Messtechnik Gmbh. The measuring system has a sensor for the shaft displacement detection, temperature and vibration sensor in the front bearing, temperature sensor in stator windings, crash sensor, tool clamping sensor and a data logger through which the monitoring system is connected to the control unit or external PC using RS485 connection.

Proper operation of the main spindle unit required design of two additional subsystems - spindle cooling system and tool/workpiece clamping aggregate. Cooling system was designed to maintain constant temperature of the main spindle unit with respect to rated power output. Hydraulic clamping aggregate, in addition to tool clamping, supports up to three cylinders for workpiece clamping.

Permanent magnet synchronous motors (PMSM), of the same type and characteristics, model 1FT5076-0AC01-Z, manufactured by Siemens AG, were used for feed drives. Motors were not selected separately, as they were already available from the stock.

Most extensive task in this part of project was design and assembly of $\mathrm{X}$ axis. This process involved replacement of a hydraulic cylinder, which previously served as the main drive, with $2500 \mathrm{~mm}$ long ball screw assembly. Suitable bearing support elements also had to be made and facing of matching areas in the machine bed had to be performed so that they could be mounted. Existing guides were originally designed for translatory main motion velocities up to $25 \mathrm{~m} / \mathrm{min}$. They were in a good condition with no visible signs of wear, for which reason they were left unchanged.

Regarding the $\mathrm{Y}$ and $\mathrm{Z}$ axis, existing drives were both replaced with suitable ball screw assemblies and corresponding belt transmissions. Existing dovetail guides were left unchanged, in order to preserve axis rigidity. Belt transmission was necessary for both axes in order to fully utilize available feed motors. Original machine design featured auxiliary vertical support drive used for rough tool height adjustment in case of higher workpiece machining requirements. At present, this drive, consisting of $\mathrm{AC}$ induction motor with worm gear and trapezoidal screw assembly, is left intact. Up to this moment, measurements that would establish machine repeatability and geometric accuracy have not yet been performed.

Existing lubrication system, originally used for the main drive, was not adequate for $\mathrm{X}$ axis guides as continuous (back and forth) full guide travel motion was required for its proper operation. Remaining guides were cord lubricated, supplied by small oil pools placed in corresponding locations, and also had to be replaced with more suitable system. Circulating oil with constant flow of $21 / \mathrm{min}$, invariant of axis travel, was required for lubrication of $\mathrm{X}$ axis guides. Impulse lubrication, cycledependent on travel distance in given period of time, was required for each remaining axis respectively. Centralized lubrication system was designed to specifically match described lubrication requirements for retrofitted feed drives.

In addition to dry machining, installation of flood/mist 
supply system, as well as suitable chip conveyors is planned in the near future. At present, automatic tool change (ATC) was not installed, although necessary interface has been provided in control system to allow possibility for later upgrade.

Design of NC operator panel aimed to provide versatile practical solution for industrial, research and educational purposes. Dual touch screens provide convenient humanmachine interface (HMI), allowing visualization of process variables in real time and user interaction through virtual control panels (VCP). In addition, standard 96-key PC keyboard with trackball mouse were installed for easier data input. Standard industrial pushbuttons were installed for control of the main spindle, clamping system, flood/mist, chip conveyer, vertical support drive, etc. Overrides for angular and linear feedrates, rapid traverse and spindle speed are available through BCD coded rotary switches, allowing 26 possible levels for each parameter. Control of up to six axis by jogging or by manual pulse generators (MPG) can be done directly from NC panel or through attached remote control.

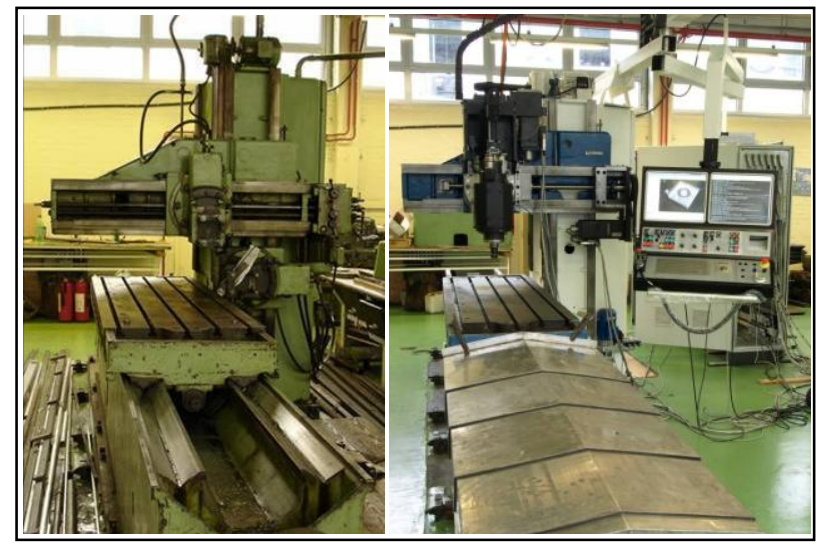

Fig. 1. Machine in its original and redesigned form

\section{CONTROL SYSTEM}

Design of control system was focused on a solution that would be as much generic as possible. This approach was used in order to provide reconfigurable system suitable for future prototype development of machine tools with various configurations. Presently, control system is capable of controlling up to six servo axis and two main spindles (control cabinet for the main spindle unit was designed separately by HSTec Co.)

Digital servo controllers type DPCANIE-030A400 are used for driving $\mathrm{Y}$ and $\mathrm{Z}$ axis motors, while DPCANIE-060A400 is used for driving $\mathrm{X}$ axis motor. Three additional controllers, type DPCANIE-015A400 were installed in order to provide possibility for feed drive upgrades.

Selected drives provide multiple modes of operation (closed loop control of position, velocity or torque, as well as encoderfollowing for electronic gearing), variety of common industrial interfaces for acquisition of reference signals $( \pm 10 \mathrm{~V}$, PWM+Direction, STEP+Direction), CAN-bus interface with CANOpen protocol for fieldbus connections, as well as multiple general purpose analog and digital I/O ports.

Interface to drives from PC side is done via suitable set of interface cards, which include both Motenc lite PCI card manufactured by Vital System Co., as well as $5 \mathrm{i} 20$ and $5 \mathrm{i} 22$ Anything IO PCI cards manufactured by Mesa Electronics Co. Compatibility with EMC2 software is provided through HostMot2 firmware.

Incremental encoders, which are powered from motor drives and utilize differential quadrature TTL output signals, are used as feedback devices for both drives and EMC. Signals from encoder are first fed to the motor drive as velocity loop feedback, buffered, and then sent to EMC.
EMC runs on Linux based operating systems with real-time extensions. This variant of OAC system has been chosen because of its high stability and software maturity in general. Up to this time, EMC has been successfully used in several CNC retrofitting projects, including applications with complex kinematic chains.

EMC is organized from four components:

- Motion Controller (EMCMOT),

- Discrete I/O Controller (EMCIO),

- Task coordinating module (EMCTASK),

- Text-based and graphical user interfaces (GUI).

EMCMOT executes cyclically in real-time and performs trajectory planning, direct and inverse kinematic calculations and computation of desired output to motor control subsystems. It interacts with the subordinate real-time modules, such as PID compensation algorithms and other hardware drivers.

EMCIO module handles all I/O functions, which are not directly related to the actual motion of machine axis. It is implemented as a single I/O controller, consisting of hierarchy of subordinate controllers for main spindle, automatic tool change, coolant, auxiliary functions (e.g., E-STOP chain, lubrication, etc.) and other user-defined subsystems.

EMCTASK is task level command handler and program interpreter for the RS-274 NGC machine tool programming language (Marietta, M., 1994), commonly referred as G code. It monitors the status of subordinate modules (EMCMOT and EMCIO) and coordinates them. It also receives and analyzes the commands, either from the operator through GUI or from another process (locally or remotely in both cases).

Several user interfaces have been developed for EMC: keystick, xemc, tkemc, mini and AXIS, which is the most advanced GUI, featuring interactive G-code previewer. More details about the EMC structure and its functionality can be found in (The EMC Team, 2009).

\section{CONCLUSION}

The three-axis milling machine presented in this paper is an industrially applicable machining system, as well as research and educational platform. It is a specific product developed using multidisciplinary knowledge and skills. Once completely finished and tested, it will be used for the analyses and implementation of process monitoring and control algorithms, which affects on machine autonomy, flexibility, and productivity. Future work will also involve upgrade of existing kinematic structure with two additional rotary axes.

\section{REFERENCES}

Asato, O.L.; Kato, E.R.R.; Inamasu, R.Y. \& Porto, A.J.V. (2002). Analysis of Open CNC Architecuture for Machine Tools, Journal of the Brazilian Society of Mechanical Sciences, Vol. 24, pp. 208-212, ISSN 0100-7386

Marietta, M., 1994, Next generation controller (NGC) specifications for an open system architecture (SOSAS) revision 2.0. Technical report, National Center for Manufacturing Sciences, Available from: ftp:// ftp.isd.mel.nist.gov/pub/NGC_document.pdf, Accessed on: 2011-06-05

Pritschow, G. et al. (2001). Open Controller Architecture Past, Present and Future, CIRP Annals - Manufacturing Technology, Vol. 50(2), pp. 463-470, ISSN 0007-8506

Proctor, F.M. \& Michaloski J. (1993), Enhanced Machine Controller Architecture Overview, Available from: ftp://ftp.isd.mel.nist.gov/pub/ NISTIR_5331.pdf, Accessed on: 2011-06-05

The EMC Team (2009). EMC User Manual, Available from: http://www.linuxcnc.org/docs/EMC2_User_Manual.pdf, Accesed on: 2009-06-06 